\title{
Plasmonic control of nonlinear two-photon absorption in graphene nanocomposites
}

\author{
Joel D. Cox ${ }^{1}$, Mahi R. Singh ${ }^{1}$, Miguel A. Antón ${ }^{2}$, and Fernando Carreño ${ }^{2}$ \\ ${ }^{1}$ Department of Physics and Astronomy, The University of Western Ontario, London N6A 3K7, Canada and \\ ${ }^{2}$ Facultad de Óptica y Optometría, Universidad Complutense de Madrid, Madrid 28037, Spain
}

\begin{abstract}
We investigate theoretically two-photon absorption in a quantum dot-graphene nanodisk nanocomposite (hybrid) system. It is considered that an external laser field is applied to the combined system which is resonant with the localized plasmons of the graphene nanodisk. The external field also excites the quantum dot via two-photon absorption. This resonance condition can be achieved by tuning the localized plasmon resonance frequency in the graphene nanodisk via electrostatic gating. We show that when the quantum dot and graphene nanodisk are in close proximity, the strong local field of the graphene plasmons can enhance and control nonlinear optical processes in the quantum dot. Specifically, we show that two-photon absorption in the quantum dot can be switched between single- or double-peaked spectra by changing the graphene-quantum dot separation. Two-photon absorption and power absorption in the quantum dot can also be switched on or off by changing the gate voltage applied to the graphene nanodisk. Our findings indicate that the present system can be used for nonlinear optical applications such as all-optical switching, biosensing and signal processing.
\end{abstract}

\section{INTRODUCTION}

Recently there has been interest in studying the enhancement of optical nonlinearities in nanocomposite systems which contain plasmonic (i.e. conducting) nanoparticles [1-11]. Typically in these types of nanocomposites, nonlinear optical processes in molecules or semiconductor nanoparticles are enhanced by the strong local electromagnetic fields created by localized surface plasmons in nearby noble metal nanoparticles [1]. A number of recent experimental and theoretical studies have demonstrated that nonlinear optical processes such as two-photon absorption/luminescence [24], second- [5, 6] and third-harmonic generation [7] in semiconductor quantum dots can be enhanced by surface plasmons in metal nanoparticles. Therefore, quantum dot-metal nanoparticle hybrid systems can be used for nonlinear optical applications such as all-optical switching, biosensing, and other types of signal processing [2]. Two-photon fluorescence from biological markers (dyes, aptamers, etc.) enhanced by metal nanoparticles has also been widely studied for applications in biological imaging [8-10]. Zhang et al. [9], for example, demonstrated energy transfer between a commonly used molecular DNA label and gold nanoparticles under two-photon excitation using fluorescence lifetime imaging microscopy. They showed that this energy transfer can be used to provide detailed information in biological studies.

Graphene has also begun to attract a great deal of attention in experimental and theoretical investigations on nanocomposite systems. In several studies, graphenequantum dot nanocomposites were fabricated and resonant energy transfer was observed through the photoluminescence of the quantum dots, which was strongly quenched in the presence of graphene [11-13]. Hybrid graphene-quantum dot phototransistors have also been fabricated recently, and were shown to exhibit ultrahigh photodetection gain with high quantum efficiency [14]. In the field of plasmonics, graphene has been recognized as a promising alternative to noble metals, as plasmons in chemically or electrostatically doped graphene have been shown to facilitate strong light-matter interactions and can be easily tuned electrostatically $[15,16]$. Strong coupling between plasmons in nanostructured graphene with a quantum emitter (i.e. quantum dot or molecule) has been predicted in theoretical studies, which could lead to the development of quantum plasmonic devices that operate at the single-photon/plasmon level [17-19]. Recently we have studied energy transfer in a quantum dotgraphene nanocomposite embedded in a photonic crystal [20].

The nonlinear optical properies of graphene-based nanocomposites have also been investigated in several experimental studies. For instance, Feng et al. [21] synthesizied graphene nanosheets decorated with tiny CdS quantum dots and observed nonlinear scattering and nonlinear absorption at two distinct excitation wavelengths. Lee et al. [22] deposited gold nanocrystals of various shapes on graphene oxide and examined the linear and nonlinear optical properties of the graphene oxide-gold nanocrystal composites. They observed a four-fold enhancement in the two-photon excitation emission intensity of the graphene oxide-gold nanocomposite compared to pure gold nanocrystals.

In the present paper we have developed a theory for the enhancement and control of two-photon absorption in a nanocomposite made by combining a doped graphene nanoflake with a quantum dot. In this hybrid system, the electrostatically tunable localized surface plasmons in the graphene nanoflake generate a strong local electromagnetic field that enhances the nonlinear optical response of the quantum dot. We show that the two-photon absorption coefficient and power absorption in the quantum dot can be controlled by changing the Fermi level in the graphene nanoflake via electrostatic gating. This effect can be used to manipulate two-photon absorption and/or fluorescence from the quantum dot. Our findings are also directly applicable to graphene nanocom- 


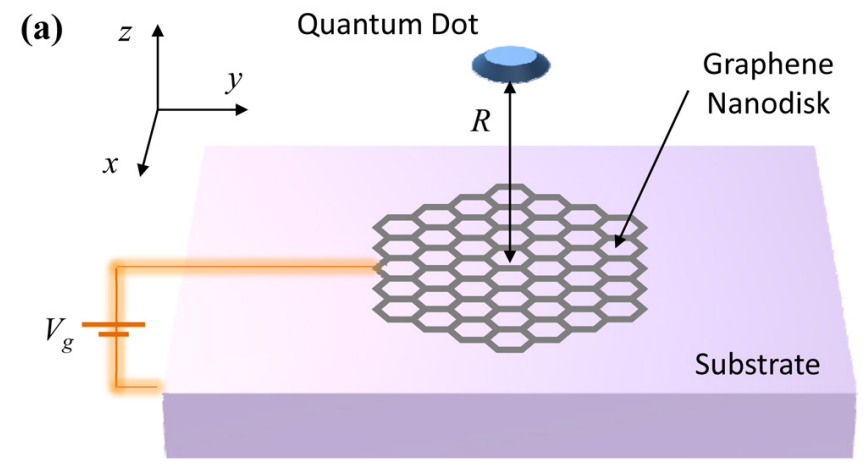

(b)
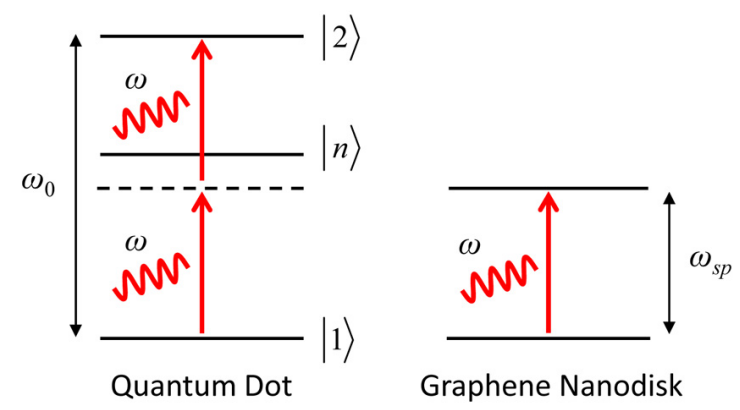

Graphene Nanodisk

FIG. 1. (a) Schematic diagram of the quantum dot-graphene nanodisk nanocomposite (hybrid) system. The graphene nanodisk lies on a dielectric substrate and a quantum dot is placed directly above at a distance $R$ from the nanodisk. The quantum dot is embedded in a dielectric medium. A gate voltage $V_{g}$ is applied to the graphene nanodisk. (b) Energy level diagrams for the quantum dot and graphene nanodisk. The external field excites surface plasmons in graphene and excitons in the quantum dot via two-photon absorption.

posites made with other types of quantum emitters such as molecules, dyes, etc. It is anticipated that the present hybrid system can be used to fabricate nonlinear optical devices such as switches, biological sensors and signal processors.

\section{LOCAL FIELD ENHANCEMENT}

In our model a semiconductor quantum dot is placed above a graphene nanoflake lying in the $x-y$ plane, where the center-to-center distance between the nanoflake and the dot is denoted as $R$. A schematic diagram of the system is shown in Fig. 1a. Together the quantum dot and graphene nanoflake form a nanocomposite or hybrid system. It is considered that the graphene nanoflake is deposited on a dielectric substrate with relative permittivity $\epsilon_{s}$, while the quantum dot is surrounded by a background dielectric material with relative permittivity $\epsilon_{b}$. An external field $E_{0} \cos (\omega t)$ is applied to the system, which interacts with both the quantum dot and graphene nanoflake.

In the graphene nanoflake the optical excitations are

localized surface plasmon polaritons, which have a resonance frequency $\omega_{s p}$. When the frequency of the external field lies near the surface plasmon resonance frequency (i.e. $\omega \approx \omega_{s p}$ ), there is a strong excitation of surface plasmons in the graphene nanoflake. These plasmons create a dipole moment $P_{g}$ in the nanoflake, which in turn produces an enhanced local field at the location of the quantum dot given as

$$
E_{g}=\frac{g_{k} P_{g}}{4 \pi \epsilon_{b} \epsilon_{0} R^{3}}
$$

Here $g_{k}(k=x, y, z)$ is called the polarization parameter, with $g_{x}=g_{y}=-1$ when the applied field is in the plane of the graphene nanoflake and $g_{z}=2$ when the applied field is perpendicular to the nanoflake. To calculate the polarization of the graphene nanoflake $P_{g}$ we use the quasistatic approximation, which allows us to assume a spatially uniform but time-varying electric field across the nanoflake. Note that this approximation is only valid for nanoparticles with dimensions much less than the wavelength of incident light [23]. An analytic expression for $P_{g}$ is obtained by modelling the graphene nanoflake as a very flat and thin oblate spheroid with semimajor axes $d_{x}=d_{y}$ and semiminor axis $d_{z}$, where $d_{x}>d_{z}$. The in-plane polarization of the nanoflake for an electric field applied in the plane of the nanoflake $(x-y$ plane) is then found as

$$
\begin{aligned}
P_{g} & =\epsilon_{s b} \epsilon_{0} \alpha_{x}(\omega) E_{0}, \\
\alpha_{x}(\omega) & =\pi d_{x}^{3} \frac{\left[\frac{\sigma_{g}(\omega)}{\epsilon_{s b} \epsilon_{0} \omega d_{x}}\right] i}{1+\frac{3 \pi}{16}\left[\frac{\sigma_{g}(\omega)}{\epsilon_{s b} \epsilon_{0} \omega d_{x}}\right] i}
\end{aligned}
$$

In the above expression, $\alpha_{x}(\omega)$ is the dipole polarizability in the two-dimensional limit $d_{x} \gg d_{z}$ [24], while the quantity $\epsilon_{s b}=\left(\epsilon_{s}+\epsilon_{b}\right) / 2$ is the effective relative permittivity of the medium surrounding the graphene nanoflake, which accounts for the differing dielectric media on either side of the nanoflake [25]. In Eq. (3), $\sigma_{g}(\omega)$ is the in-plane conductivity of graphene and is obtained as [17]

$$
\begin{aligned}
\sigma_{g}(\omega) & =\frac{e^{2} E_{F}}{\pi \hbar^{2}} \frac{i}{\omega+i \tau^{-1}} \\
& +\frac{e^{2}}{4 \hbar}\left[\theta\left(\hbar \omega-2 E_{F}\right)+\frac{i}{\pi} \log \left|\frac{\hbar \omega-2 E_{F}}{\hbar \omega+2 E_{F}}\right|\right]
\end{aligned}
$$

Here $E_{F}$ is the Fermi energy of the doped graphene nanoflake and $\tau=\mu E_{F} / e v_{F}^{2}$ is the intrinsic relaxation time, where $v_{F}$ is the Fermi velocity and $\mu$ is the dc mobility. At the surface plasmon resonance frequency $\omega_{s p}$, the real part of the denominator in Eq. (3) becomes zero and $\alpha_{x}$ becomes large. Therefore, the local field from the graphene nanoflake $\left(E_{g}\right)$ is strongest when $\omega=\omega_{s p}$.

In a number of experimental studies it has been shown that the Fermi energy in graphene can be controlled via electostatic gating, where the relationship between $E_{F}$ and an external gate voltage $V_{g}$ can be described using a 
simple capacitor model as [26, 27]

$$
\left|E_{F}\right|=\hbar v_{F} \sqrt{\pi C_{g}\left|V_{g}+V_{0}\right|}
$$

In the above expression, $C_{g}$ is a constant which depends on the gate capacitance and $V_{0}$ is the offset voltage caused by natural doping. Hence, the Fermi energy can be controlled by the external gate voltage. This means that in the graphene nanoflake the polarizability $\alpha_{x}(\omega)$ and surface plasmon resonance frequency $\omega_{s p}$, which depend on the Fermi energy, can be tuned by varying the gate voltage.

Since the quantum dot is near the graphene nanoflake it interacts with both the external field and the local field produced by the graphene nanoflake, both of which oscillate at frequency $\omega$. The total electric field experienced by the quantum dot is then given from

$$
E_{q d}=\frac{1}{2 \epsilon_{e f f}}\left(E_{0}+E_{g}\right) e^{-i \omega t}+c . c .
$$

where $\epsilon_{e f f}=\left(2 \epsilon_{b}+\epsilon_{q d}\right) / 3 \epsilon_{b}, \epsilon_{q d}$ being the dielectric constant of the quantum dot. We treat the quantum dot as a two-level system, where $|1\rangle$ and $|2\rangle$ are the ground and excited states, respectively, and the resonant frequency for the transtion $|1\rangle \leftrightarrow|2\rangle$ is denoted by $\omega_{0}$. In this paper we consider the case where the two-photon resonance frequency of the quantum dot is twice the surface plasmon resonance frequency of the graphene nanoflake, i.e. $\omega_{0}=2 \omega_{s p}$ (see Fig. 1b). Due to the strong local field from the graphene nanoflake, nonlinear two-photon absorption occurs in the quantum dot when $2 \omega=\omega_{0}$. In our model, the quantum dot undergoes two-photon excitation from the ground state $|1\rangle$ to excited state $|2\rangle$ through virtual transitions involving one or more intermediate states $|n\rangle$ which are far from resonance with the external field. In this case the polarization of the quantum dot $P_{q d}$ can be expressed as [28]

$$
\begin{aligned}
P_{q d} & =\left(k_{11} \rho_{11}+k_{22} \rho_{22}\right)\left(\frac{E_{0}}{\epsilon_{e f f}}+\frac{E_{g}}{\epsilon_{e f f}}\right) \\
& +2 k_{21}\left(\frac{E_{0}}{\epsilon_{e f f}}+\frac{E_{g}}{\epsilon_{e f f}}\right)^{*} \rho_{21}
\end{aligned}
$$

In the above expression, $\rho_{i j}(i, j=1$ or 2$)$ are the twolevel density matrix elements which are obtained in the following section of this paper, and $k_{i j}$ are called the two-photon coefficients and are given as

$$
\begin{aligned}
& k_{21}=\frac{1}{\hbar} \sum_{n} \frac{\mu_{2 n} \mu_{n 1}}{\omega_{n 1}-\omega} \\
& k_{i i}=\frac{2}{\hbar} \sum_{n} \frac{\left|\mu_{n i}\right|^{2} \omega_{n i}}{\omega_{n i}^{2}-\omega^{2}}
\end{aligned}
$$

Here $\omega_{n i}$ and $\mu_{n i}$ and the transition frequencies and dipole matrix elements, respectively, between states $|n\rangle$ and $|i\rangle$. The first and second terms in Eq. (7) represent the contributions to the polarization of the quantum dot from the off-resonant dipoles (which depends on the populations in states $|1\rangle$ and $|2\rangle$ given by $\rho_{i i}$ ) and the two-photon two-level coherence $\rho_{21}$, respectively $[28,29]$.

\section{DENSITY MATRIX FORMALISM}

The density matrix elements appearing in Eq. (7) are evaluated from their equations of motion obtained using the master equation method [28, 30]. Using Eq. (6), the two-level two-photon density matrix equations of motion are obtained as

$$
\begin{aligned}
\frac{d \rho_{22}}{d t} & =-\Gamma \rho_{22}+i \Omega_{0}\left(1+\Pi_{g}\right)^{2} \rho_{12} \\
& -i \Omega_{0}\left(1+\Pi_{g}^{*}\right)^{2} \rho_{21} \\
\frac{d \rho_{12}}{d t} & =-\left[\gamma-i\left(\Delta+\Delta_{S}\right)\right] \rho_{12} \\
& +i \Omega_{0}\left(1+\Pi_{g}^{*}\right)^{2}\left(\rho_{22}-\rho_{11}\right)
\end{aligned}
$$

where

$$
\begin{aligned}
\Delta_{S} & =\beta_{S} \Omega_{0}\left|1+\Pi_{g}\right|^{2}, \\
\Omega_{0} & =\frac{k_{21} E_{0}^{2}}{2 \hbar \epsilon_{e f f}^{2}}, \Pi_{g}=\frac{g_{x} \alpha_{x}(\omega)}{4 \pi R^{3}}
\end{aligned}
$$

Here $\Delta=2 \omega-\omega_{0}$ is the two-photon detuning parameter, $\Gamma$ and $\gamma$ are the relaxation and dephasing rates, respectively, for the transition $|1\rangle \leftrightarrow|2\rangle$, and $\beta_{S}=$ $\left(k_{11}-k_{22}\right) /\left(2 k_{21}\right)$ [28]. The equations of motion for the remaining density matrix elements are easily obtained through the relations $\rho_{11}+\rho_{22}=1$ and $\rho_{12}=\rho_{21}^{*}$. In the above expressions, the parameter $\Omega_{0}$ denotes the twophoton Rabi frequency due to direct two-photon absorption in the quantum dot from the external field, while the term $\Pi_{g}$ represents the local field enhancement factor due to the plasmons excited in the graphene nanoflake. Finally, the parameter $\Delta_{S}$ represents the dynamic Stark shift which appears due to frequency shifts in the levels $|1\rangle$ and $|2\rangle$ induced by virtual transitions to the intermediate level(s) $|n\rangle[28,29]$. The density matrix equations of motion are solved under steady-state conditions to obtain the elements $\rho_{i j}$, which are used to calculate $P_{q d}$.

\section{TWO-PHOTON ABSORPTION}

The quantum dot absorbs energy from the external field and from the enhanced local field of the graphene nanoflake through a two-photon absorption process. The two-photon absorption coefficient of the quantum dot can be obtained from $P_{q d}$ as [28]

$$
\alpha_{T P A}=\operatorname{Re}\left(-\frac{i \omega}{2 \epsilon_{b} \epsilon_{0} c V_{q d}} \frac{P_{q d}}{E_{q d}}\right)
$$

where $V_{q d}$ is the volume of the quantum dot. Analytical expressions for the density matrix elements $\rho_{i j}$ are obtained in the steady-state and used in Eq. (7) to obtain 
the two-photon absorption coefficient of the quantum dot as

$$
\alpha_{T P A}=\frac{\omega k_{21}}{\epsilon_{b} \epsilon_{0} c V_{q d}} \frac{\gamma \Gamma \Omega_{0}\left|1+\Pi_{g}\right|^{2}}{\Gamma\left[\gamma^{2}+\left(\Delta+\Delta_{S}\right)^{2}\right]+4 \gamma \Omega_{0}^{2}\left|1+\Pi_{g}\right|^{4}}
$$

Notice that the above expression depends on both the external field $\left(\Omega_{0}\right)$ and the local field produced by the graphene nanoflake $\left(\Pi_{g}\right)$. The second term in the denominator leads to a broadening of the absorption spectrum that depends on the intensity of the external field through $\Omega_{0}$. This broadening is referred to as power broadening in the literature [31]. However, the second term in the denominator also depends on the local field from the graphene nanoflake through the term $\Pi_{g}$, which depends on the graphene polarizability $\alpha_{x}(\omega)$ and the center-tocenter distance between the nanoflake and the quantum $\operatorname{dot} R$. This is an interesting effect which we call local field broadening. We also note that the two-photon absorption coefficient is inversely related to $\Omega_{0}$ and $\Pi_{g}$.

Following the method of Ref. [20] we also calculate the power absorbed by the quantum dot via the two-photon absorption process as

$$
W_{q d}=\hbar \omega_{0} \Gamma \rho_{22}
$$

Using the steady-state solution for $\rho_{22}$ we obtain

$$
W_{q d}=\frac{2 \hbar \omega_{0} \Gamma \gamma \Omega_{0}^{2}\left|1+\Pi_{g}\right|^{4}}{\Gamma\left[\gamma^{2}+\left(\Delta+\Delta_{S}\right)^{2}\right]+4 \gamma \Omega_{0}^{2}\left|1+\Pi_{g}\right|^{4}}
$$

Note that the power absorbed by the quantum dot depends on the population density $\rho_{22}$ of the excited state $|2\rangle$, which in turn depends on the local field enhancement from the graphene nanoflake through $\Pi_{g}$. This shows that energy absorbed by the graphene nanoflake can be transfered to the quantum dot. However, there is no energy transfer from the quantum dot to the graphene nanoflake because conservation of energy would not be satisfied in this process ( since $\omega_{0} \neq \omega_{s p}$ ).

\section{RESULTS AND DISCUSSION}

The graphene nanoflake has a radius of $d_{x}=17 \mathrm{~nm}$ and is deposited on a silica substrate $\left(\epsilon_{s}=1.96\right)$. To calculate the optical response of the graphene nanoflake we use $C_{g}=2 \times 10^{16} \mathrm{~m}^{-2} \mathrm{~V}^{-1}$ and $V_{g}+V_{0}=36.6 \mathrm{~V}$, while $v_{F}=c / 300$ is the Fermi velocity and $\mu=10000$ $\mathrm{cm}^{2} \mathrm{~V}^{-1} \mathrm{~s}^{-1}$ is the dc mobility [17]. The background dielectric material for the hybrid system is taken as GaAs $\left(\epsilon_{b}=10.95\right)$. Using the parameters given above, the surface plasmon resonance frequency of the graphene nanoflake is calculated using Eq. (3) as $\omega_{s p}=175 \mathrm{meV}$. As a realistic example, we choose a p-type InAs/GaAs self-assembled quantum dot $\left(\epsilon_{q d}=11.84\right)$ as described in Ref. [32]. For this quantum dot we take the energy difference for the transition from the ground state $|1\rangle$ to the intermediate state $|n\rangle$ as $\hbar \omega_{n 1}=160 \mathrm{meV}$, while the two-photon resonance frequency and relaxation rate are $\hbar \omega_{0}=350 \mathrm{meV}$ and $\hbar \Gamma=200 \mu \mathrm{eV}$, respectively [32]. Note that the p-type InAs/GaAs quantum dot was chosen so that the resonance frequencies of the graphene nanoflake and quantum dot satisfy the condition $2 \omega_{s p}=\omega_{0}$. The in-plane transition dipole moments are obtained in Ref. [32] as $\mu_{n 1}=0.11 \mathrm{e} \times \mathrm{nm}$ and $\mu_{2 n}=0.21 \mathrm{e} \times \mathrm{nm}$. In the following simulations we consider that the external driving field is polarized along the $x$-axis and has an intensity of $750 \mathrm{~kW} / \mathrm{cm}^{2}$.

We first study the effect of the separation distance between the quantum dot and graphene nanoflake on twophoton processes in the quantum dot. In Fig. 2 we plot the two-photon absorption coefficient $\left(\alpha_{T P A}\right)$ as a function of the two-photon detuning $\Delta$ for various values of quantum dot-graphene nanoflake separation $R$.

In Fig. 2a we find that when the separation is larger (i.e. $R=40 \mathrm{~nm}$ or higher) the absorption spectrum consists of a single, narrow peak located at $\Delta=0$. As $R$ is decreased to 30 and $15 \mathrm{~nm}$ (dashed and dash-dotted curves, respectively), the absorption spectrum broadens and the peak height decreases. This broadening is a result of the local field broadening as discussed in Eq. (14). The asymmetry in the two-photon absorption spectrum is a result of the dynamic two-photon Stark shift $\Delta_{S}$, which also depends on the local field enhancement factor $\Pi_{g}$ and increases as $R$ decreases (see Fig. 2a inset).

In Fig. $2 \mathrm{~b}$ we show the two-photon absorption spectrum of the quantum dot when considering smaller values of the graphene-quantum dot separation $R$. In this case the absorption spectrum shifts from a single narrow peak at $R=30 \mathrm{~nm}$ (solid curve) to two local field-broadened peaks near $\Delta= \pm 3 \mathrm{meV}$ and a minimum at $\Delta=0$ when $R=15 \mathrm{~nm}$ (dash-dotted curve). The observed splitting in the absorption spectrum is due to the local field enhancement term $\Pi_{g}$ in Eq. (14). This term depends on the detuning of the external field from the plasmon resonance in graphene through the polarizability $\alpha_{x}(\omega)$. The local field enhancement term has a maximum at $\Delta=0$, where the external field is resonant with the plasmons in graphene (i.e. $\omega=\omega_{s p}$ ), and increases as $R$ decreases (see Fig. 2b inset). Therefore, for sufficiently small values of $R$, the local field from the graphene nanoflake $\left(E_{g}\right)$ is large enough to suppress two-photon absorption in the quantum dot at frequencies near $\Delta=0$. This phenomenon is evident from Eq. (14), which shows that $\alpha_{T P A}$ tends to zero as $\left|\Pi_{g}\right|$ becomes very large.

The quantum dot power absorption spectrum given from Eq. (16) is plotted in Fig. 3(a). We find that for larger quantum dot-graphene separation values (i.e. $R=40 \mathrm{~nm}$ ) the power absorption spectrum has a single, narrow peak. The width of the power absorption peak drastically increases as $R$ is decreased to $R=30 \mathrm{~nm}$ due to the local field broadening as explained in Fig. 2a. The height of the power absorption peak also increases as $R$ is decreased. A further decrease of the distance to $R=15$ $\mathrm{nm}$ results in the emergence of a two-peaked structure 

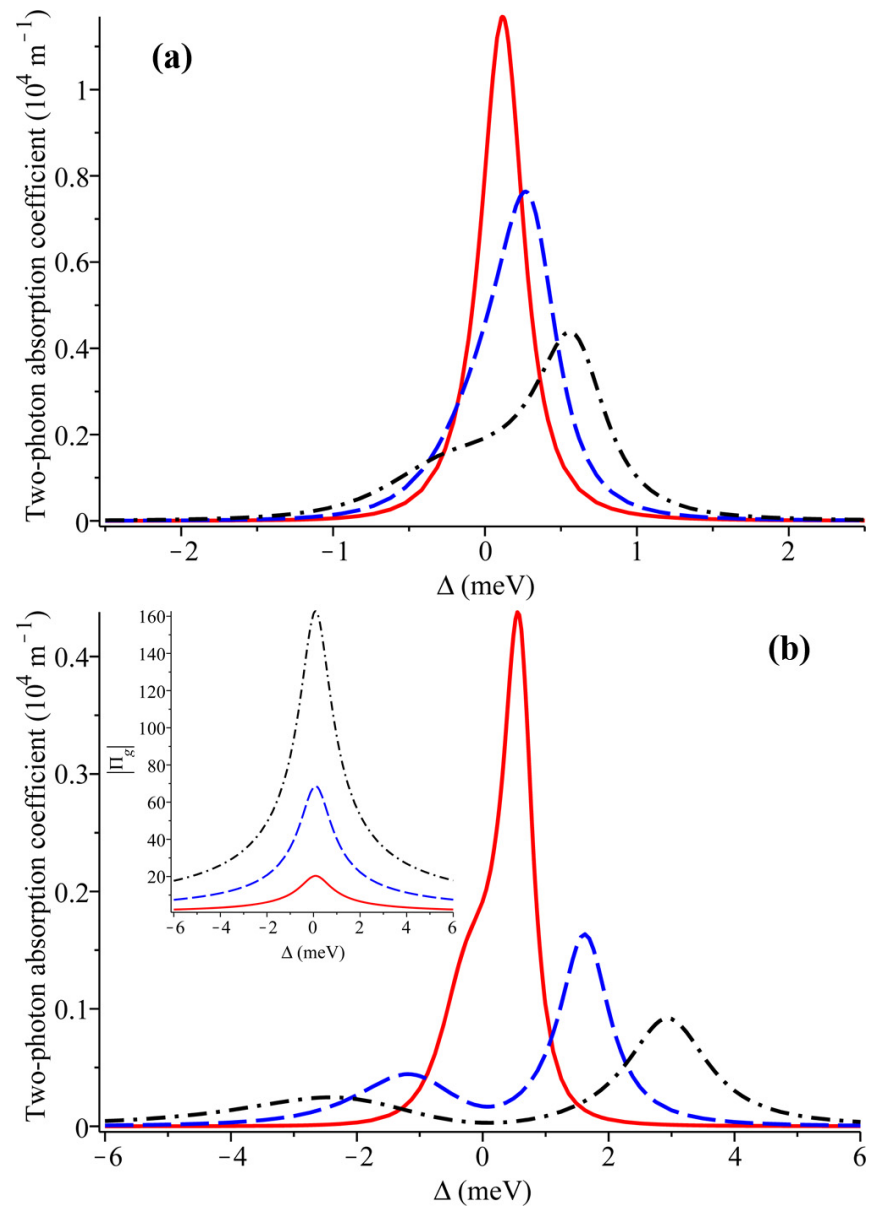

FIG. 2. Two-photon absorption coefficient $\left(\alpha_{T P A}\right)$ vs. twophoton detuning $(\Delta)$ for different values of the graphenequantum dot separation $R$. Here the gate voltage in graphene is held fixed so that $V_{0}+V_{g}=36.6 \mathrm{~V}$. (a) $R=40 \mathrm{~nm}$ (solid curve), $R=35 \mathrm{~nm}$ (dashed curve), and $R=30 \mathrm{~nm}$ (dashdotted curve). (b) $R=30 \mathrm{~nm}$ (solid curve), $R=20 \mathrm{~nm}$ (dashed curve) and $R=15 \mathrm{~nm}$ (dash-dotted curve). Inset: Local field enhancement factor $\left|\Pi_{g}\right|$ as a function of $\Delta$ for $R=30 \mathrm{~nm}$ (solid curve), $R=20 \mathrm{~nm}$ (dashed curve), and $R=15 \mathrm{~nm}$ (dash-dotted curve).

in the power absorption spectrum, and we observe two maxima (points $\mathrm{A}$ and $\mathrm{C}$ ) and a local minimum (point B). This behavior arises from the competition between the local field enhancement of the Rabi frequency and the Stark shift which is also enhanced by the plasmon interaction. In effect, let us consider the equation of motion of the density matrix (10): we can devise that the effective Rabi frequency which drives the $\mathrm{QD}$ is given by $\Omega_{e f f} \equiv \Omega_{0}\left(1+\Pi_{g}^{*}\right)^{2}$, while the effective detuning is given by $\Delta_{\text {eff }} \equiv\left(\Delta+\Delta_{S}\right)$. We should remind here that $\Delta_{S}$ is a negative magnitude since $\Omega_{0}<0$, thus the system will be close to resonance in the situation which minimizes $\Delta_{\text {eff }}$ while keeping a value of $\Omega_{\text {eff }}$ high enough to drive efficientyly the QD from the ground level to the upper level through the two-photon step. These two com-
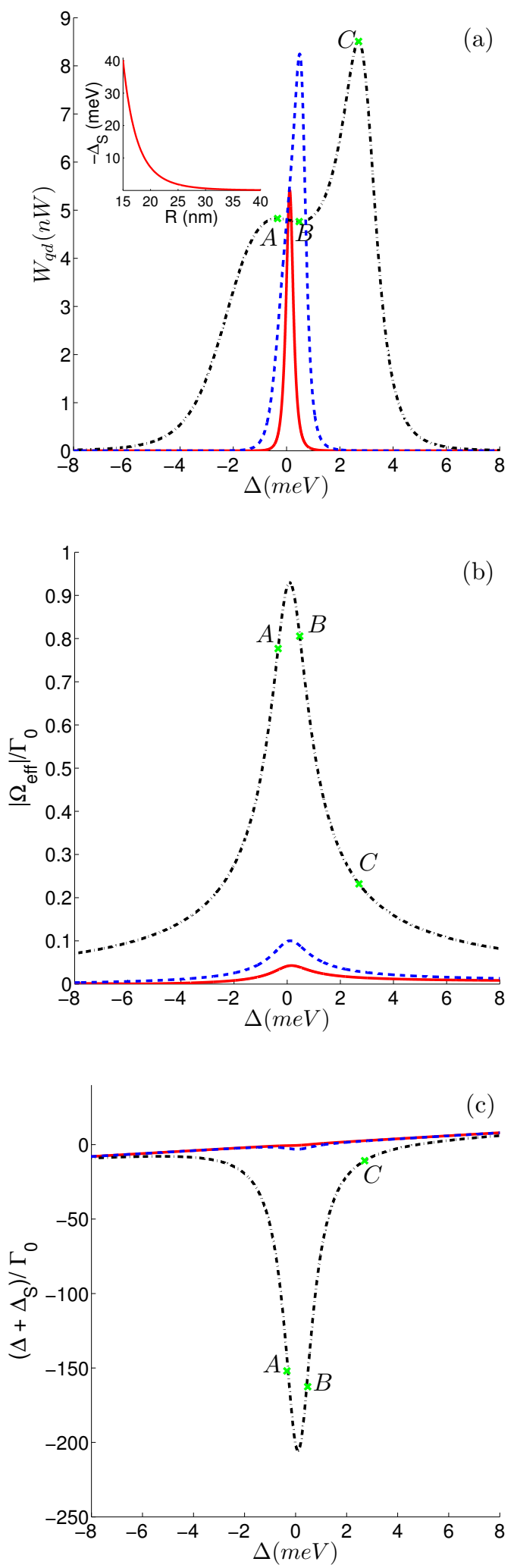

FIG. 3. (a) Power absorption in the quantum dot $\left(W_{q d}\right)$ vs. two-photon detuning $(\Delta)$ for cases where the graphenequantum dot separation is $R=40 \mathrm{~nm}$ (solid curve), $R=30$ $\mathrm{nm}$ (dashed curve), and $R=15 \mathrm{~nm}$ (dash-dotted curve). Here we take $V_{0}+V_{g}=36.6 \mathrm{~V}$. Inset: Stark shift parameter $\Delta_{S}$ as a function of $R$. (b) Effective Rabi frequency $\Omega_{e f f}$ normalized to $\Gamma_{0}$ vs. two-photon detuning $(\Delta)$. (c) Effective detuning $\Delta+\Delta_{S}$ normalized to $\Gamma_{0}$ vs. two-photon detuning $(\Delta)$. In (b) and (c) we use the same labels for the distances considered in (a). 
peting effects produced by the plasmon interaction balance each other to produce the two maxima at points $\mathrm{A}$ and $\mathrm{C}$, while at point $\mathrm{B}$ the effect of the Stark shift $\Delta_{S}$ dominates, and it results in a reduced value of the power transfer to the QD. This can be inferred by considering the $\Delta$-depencence of both magnitudes $\left(\Omega_{e f f}\right.$ and $\left.\Delta_{e f f}\right)$ presented in panels (b) and (c) of Fig. 3. The global maximum at point $C$ is obtained when magnitude $\Delta_{e f f}$ is minimum (see panel (c)) while the effective Rabi frequency remains appreciable (see panel (b)). The amount of power transferred at point $\mathrm{A}$ is obtained for a point where the effective detuning reach a large value: in a conventional two-level system this situation will result in a negligible population of the upper-level. However, in the current system the effective Rabi frequency is also $\Delta$-dependent and exhibits a large enhancement which allows for a large population of the upper level even in the presence of such a large value of $\Delta_{e f f}$ at point A.

For the hybrid system we have considered, the value of the graphene-quantum dot separation $R$ can be controlled by changing the thickness of the GaAs between the graphene nanoflake and the quantum dot during fabrication. Once the system is fabricated, the separation can be changed by applying stress and strain fields (i.e. the hybrid system can serve as a pressure sensor). The results we have presented here are also valid if the quantum dot is replaced by a chemical or biological molecule for which two-photon absorption occurs. In this case the hybrid system can be used as a biological sensor, where different molecules attached to the graphene nanoflake will have different effective separations $R$. This system can also be used as a thermal sensor by using a thermally sensitive spacing material between the quantum emitter and graphene. As the temperature increases the thermal spacer will expand, thereby changing $R$.

We now investigate the effect of the gate voltage in graphene on the two-photon processes in the quantum dot. Recent studies have shown that plasmons in graphene can be conveniently controlled using electrostatic gating [15-19]. By changing the gate voltage $V_{g}$ applied to the graphene nanoflake, one can change the concentration of conducting electrons in the nanoflake. This changes the Fermi energy in graphene through Eq. (5), which in turn changes the plasmon resonance frequency of the nanoflake through Eqs. (4) and (3). To consider the effect of the gate voltage we replace $V_{g}$ in Eq. (5) by $V_{g}+\Delta V_{g}$ and vary $\Delta V_{g}$. Note that here the applied gate voltage does not alter the energy levels of the quantum dot, but merely modifies the properties of the graphene nanoflake.

The two-photon absorption coefficient of the quantum dot is plotted in Fig. 4 as a function of the two-photon detuning for various values of $\Delta V_{g}$. Here we have taken the graphene-quantum dot separation as $R=15 \mathrm{~nm}$. The solid curve in Fig. 3a corresponds to the dash-dotted curve in Fig. 2b, where $\Delta V_{g}=0$ and a minimum appears at $\Delta=0$. By increasing the gate voltage such that $\Delta V_{g}=1.0$ and $2.0 \mathrm{~V}$ (see dashed and dash-dotted

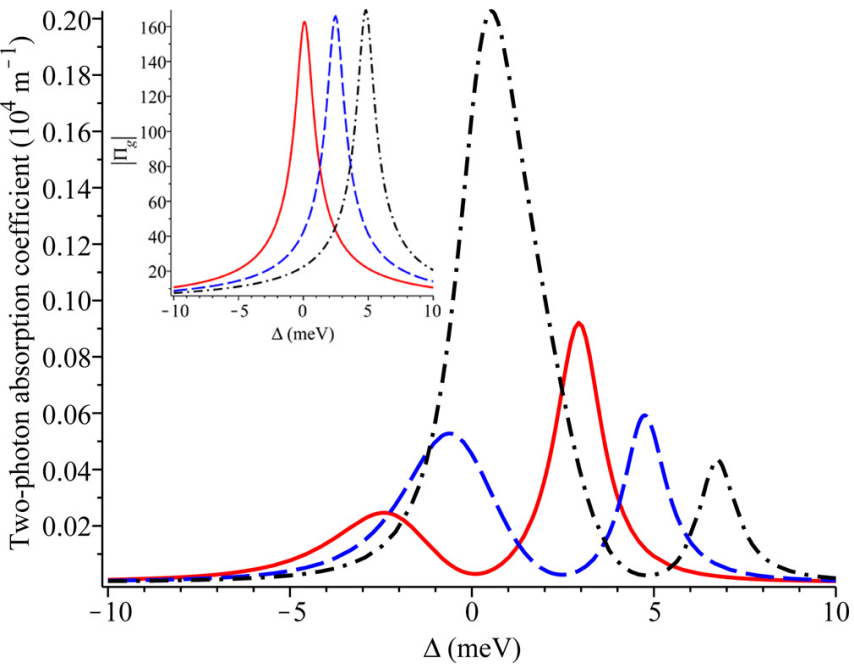

FIG. 4. Two-photon absorption coefficient $\left(\alpha_{T P A}\right)$ vs. twophoton detuning $(\Delta)$ for cases where $\Delta V_{g}=0$ (solid curve), $\Delta V_{g}=1.0 \mathrm{~V}$ (dashed curve), and $\Delta V_{g}=2.0 \mathrm{~V}$ (dash-dotted curve). Here we have maintained $V_{g}+V_{0}=36.6 \mathrm{~V}$ and $R=15$ $\mathrm{nm}$. Inset: Local field enhancement factor $\left(\Pi_{g}\right)$ as a function of the two-photon detuning when $\Delta V_{g}=0$ (solid curve), $\Delta V_{g}=1.0 \mathrm{~V}$ (dashed curve), and $\Delta V_{g}=2.0 \mathrm{~V}$ (dash-dotted curve).

curves, respectively, in Fig. 4) we find that the minimum in the absorption spectrum shifts towards positive detunings. This occurs due to the shift in the plasmon resonance frequency of the graphene nanoflake, which changes from $\omega_{s p}=175 \mathrm{meV}$ to $\omega_{s p}^{g}=\omega_{s p}+1.24 \mathrm{meV}$ and $\omega_{s p}^{g}=\omega_{s p}+2.41 \mathrm{meV}$ when $\Delta V_{g}=1.0$ and $2.0 \mathrm{~V}$, respectively. The change in the plasmon frequency changes the frequency at which $\left|\Pi_{g}\right|$ has its maximum value (see Fig. 4 inset). It is also worth pointing out that the local field enhancement factor decreases substatially at $\Delta=0$ as the gate voltage changes from $\Delta V_{g}=0$ to $\Delta V_{g}=2.0 \mathrm{~V}$ (i.e. the peak in $\left|\Pi_{g}\right|$ is shifted away from $\Delta=0$ ). When the gate voltage is increased the enhancement factor has a very small value at $\Delta=0$ and the two-photon absorption coefficient switches from a minimum to a maximum value.

From the above discussion we conclude that the minimum in the two-photon absorption spectrum can be shifted by changing the applied gate voltage. The secondary peaks in the absorption spectrum at higher detunings (i.e. at $\Delta \approx 5 \mathrm{meV}$ when $\Delta V_{g}=1.0 \mathrm{~V}$ or at $\Delta \approx 7 \mathrm{meV}$ when $\Delta V_{g}=2.0 \mathrm{~V}$ ) are weaker because the external field is significantly detuned from the twophoton resonance of the quantum dot. By applying the external gate voltage to the hybrid system, these secondary absorption peaks can also be switched from high to low values.

Finally in Fig. 5 we have plotted the two-photon power absorption spectrum when $R=25 \mathrm{~nm}$ for cases where $\Delta V_{g}=0,1.5$ and $3.0 \mathrm{~V}$ (see solid, dashed and dashdotted curves, respectively). Note that the peak in the 


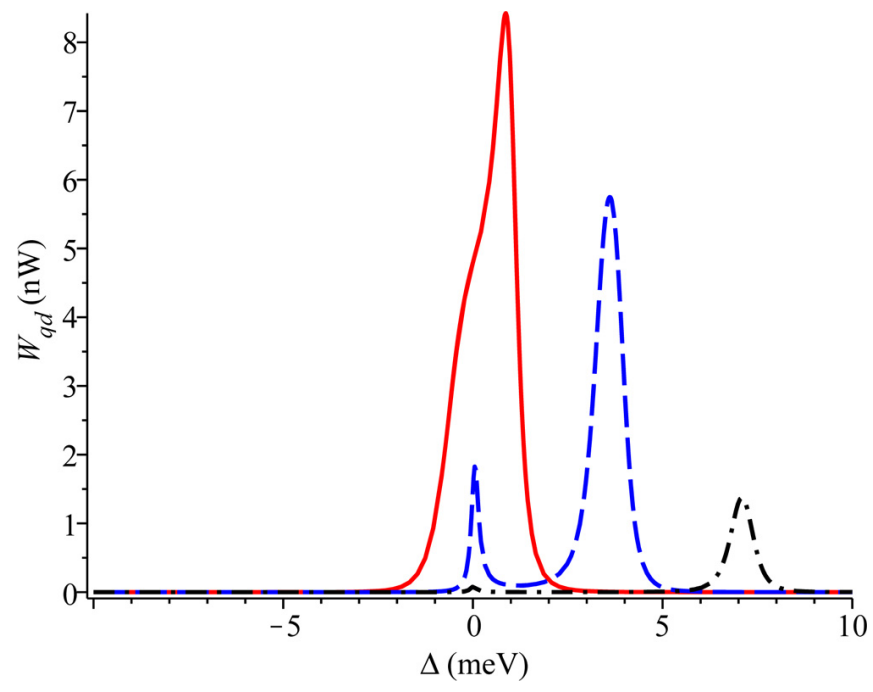

FIG. 5. Power absorption in the quantum dot $\left(W_{q d}\right)$ vs. twophoton detuning $(\Delta)$ when the gate voltage in graphene is changed so that $\Delta V_{g}=0$ (solid curve), $\Delta V_{g}=1.5 \mathrm{~V}$ (dashed curve), and $\Delta V_{g}=3.0 \mathrm{~V}$ (dash-dotted curve). Here we keep $V_{0}+V_{g}=36.6 \mathrm{~V}$ and $R=25 \mathrm{~nm}$.

power absorption spectrum shifts away from $\Delta=0$ towards positive detuning as the gate voltage is increased. This means that at $\Delta=0$ the power absorption switches from high to very low values as $\Delta V_{g}$ is increased only slightly. This result is again due to the change in the plasmon resonance of the graphene nanoflake as the gate voltage is increased. As the plasmon resonance is shifted to new frequencies, the local field enhancement factor $\Pi_{g}$ is shifted accordingly (see Fig. 4 inset). The peak in the power absorption spectrum corresponds to the location of the maximum in $\left|\Pi_{g}\right|$ (i.e. the new plasmon resonance $\left.\omega_{s p}^{g}\right)$.

Now we resort to analyze how the size of the nanodisk influences the power transfer to the QD. This question is pertinent in view of the fact that the plasmon resonance can be roughly stimated to be given by $\omega_{s p}=\sqrt{3 e^{2} E_{F} / 16 \hbar^{2} \epsilon_{s b} \epsilon_{0} d_{x}}$. This condition points out explicitely such dependence on $d_{x}$. In order to analyze the effect of the graphene flake size on $W_{q d}$, we carried out numerical simulations by slightly changing the value of $d_{x}$ around the original value of $17 \mathrm{~nm}$ while keeping fixed the intensity of the driving field $\left(I_{0}=750 \mathrm{~kW} / \mathrm{cm}^{2}\right)$ and the value of $R=15 \mathrm{~nm}$. The results are depicted in Figure 6(a) for $d_{x}=16 \mathrm{~nm}$ and $d_{x}=18 \mathrm{~nm}$.

In the case of the largest value $\left(d_{x}=18 \mathrm{~nm}\right.$, dasheddotted line) the power transferred to the QD exhibit a two-peaked structure: one of the peak is located close to $\Delta=0$ whereas the other shifts to the negative detuning region. For this size of the nanodisk, the new plasmon resonance $\omega_{s p}$ falls downward to $170.8 \mathrm{meV}$, which in turn results in a shift of the resonance for the local field enhancement factor $\Pi_{g}$ to the negative detuning region. In the case of the shortest value considered in in panel (a)
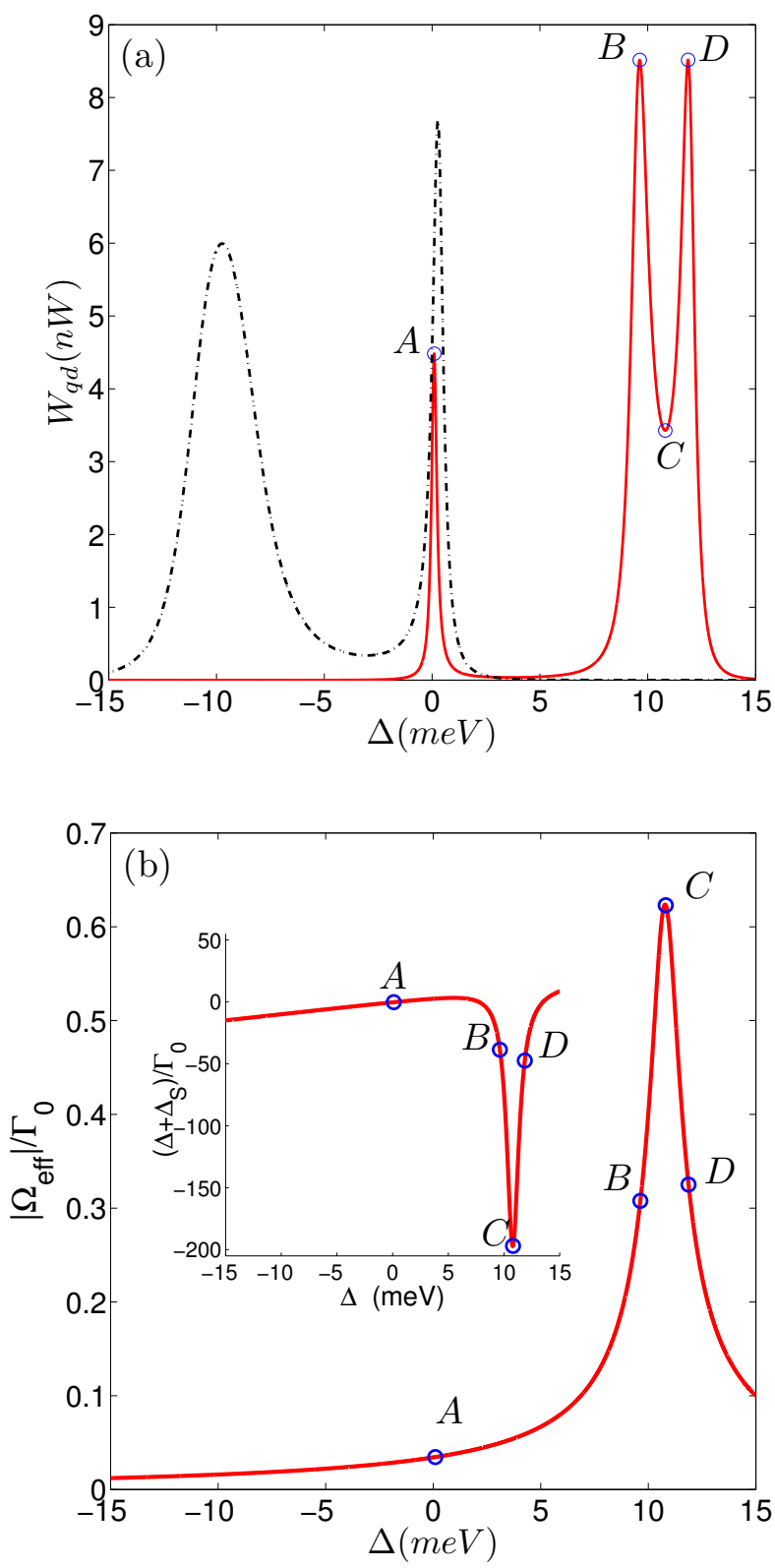

FIG. 6. (a) Power absorption in the quantum dot $\left(W_{q d}\right)$ vs. two-photon detuning $(\Delta)$ for cases where the size of the graphene nanodisk is $d_{x}=16 \mathrm{~nm}$ (solid line) and $d_{x}=18 \mathrm{~nm}$ (dashed-dotted line). (b) Effective Rabi frequency $\Omega_{\text {eff }}$ normalized to $\Gamma_{0}$ vs. two-photon detuning $(\Delta)$ for the case with $d_{x}=16 \mathrm{~nm}$. Inset: effective detuning $\Delta+\Delta_{S}$ normalized to $\Gamma_{0}$ vs. two-photon detuning $(\Delta)$. Other values are $R=15$ $\mathrm{nm}$ and the rest of parameters as in Figure 3.

$\left(d_{x}=16 \mathrm{~nm}\right.$, solid line), the power transferred to the QD exhibit a three-peaked structure: we recover a peak close to $\Delta=0$ (point A) and two outer maxima (points B and D) with similar height and a local minimum (point C) is obtained between these two maxima. For this particular size of the nanodisk, the new plasmon resonance $\omega_{s p}$ rises 
upward to $181.1 \mathrm{meV}$, which in turn results in a shift of the resonance for the local field enhancement factor $\Pi_{g}$ to the positive detuning region. The split of the expected maximum into two maxima (points B and D in Fig. 6(a)) arises again from the competition between the dynamical Stark shift and the local field enhancement of the Rabi frequency, in a similar way to the results obtained for the dashed-dotte line in Fig. 3(a). This can be further confirmed by inspecting panel (b) of Figure 6 which depicts the effective Rabi frequency and the effective detuning: the Stark shift at poinst $\mathrm{C}$ is the maximum (see the inset Figure 6(b)), and simultaneously, the enhancement of the Rabi frequency achieves the maximum. Thus, at point $\mathrm{C}$ the Stark shift dominates over the enhancement of Rabi frequency, whereas at points B and D the latter dominates and produces the maxima.

\section{CONCLUSIONS}

We have theoretically investigated two-photon absorption in a quantum dot-graphene nanoflake nanocomposite system. It is found that when the frequency of the external laser field coincides with the plasmon resonance of the graphene nanoflake, a strong local field is generated by the plasmons in the nanoflake. When the quantum dot is near the nanoflake, the local field from graphene causes a broadening of the two-photon absorption spectrum. As the quantum dot is brought very close to the nanoflake, the spectrum of two-photon absorption splits from a single peak to two peaks. It is also found that power absorption in the quantum dot is enhanced by the local field from the graphene nanoflake. We have also shown that for this nanocomposite system the two-photon absorption process in the quantum dot can be further manipulated by changing the Fermi energy in the graphene nanoflake via electrostatic gating. Our findings indicate that twophoton absorption and power absorption in the quantum dot can be switched on or off by changing the gate voltage across the graphene nanoflake. These results suggest that the quantum dot-graphene nanoflake hybrid system can be used for nonlinear optical applications such as all-optical switching, biosensing and signal processing.

\section{ACKNOWLEDGMENTS}

J. D. Cox is grateful to the Ontario Graduate Scholarship program for financial support in the form of a research scholarship. M. R. Singh wishes to thank the Natural Sciences and Engineering Research Council (NSERC) of Canada for financial support in the form of a research grant. M. A. Antón and F. Carreño acknowledge financial support from Project no. FIS2010-22082 (MCINN) from Spain.
[1] M. Achermann, J. Phys. Chem. Lett. 1, 2837-2843 (2010).

[2] M. Fu, K. Wang, H. Long, G. Yang, P. Lu, F. Hetsch, A. S. Susha, and A. L. Rogach, Appl. Phys. Lett. 100, 063117 (2012).

[3] S. Xiao, H. Gong, X. Su, J. Han, Y. Han, M. Chen, and Q. Wang, J. Phys. Chem. C 111, 10185-10189 (2007).

[4] X. Li, F. -J. Kao, C. -C. Chuang, and S. He, Opt. Express 18,11335 (2010).

[5] E. Shaviv and U. Banin, ACS Nano 4, 1529-1538 (2010).

[6] P. M. Jais, C. von Bilderling, and A. V. Bragas, Papers in Physics 3, 030002 (2011).

[7] X. Feng, Y. Chen, and D. Hou, Physica B 406, 1702-1705 (2011).

[8] N. J. Durr, T. Larson, D. K. Smith, B. A. Korgel, K. Sokolov, and A. Ben-Yakar, Nano Lett. 7 941-945 (2007).

[9] Y. Zhang, D. J. S. Birch, and Y. Chen, Appl. Phys. Lett. 99103701 (2011).

[10] A. Ray, Y.-E. K. Lee, G. Kim, and R. Kopelman, Small 8, 2213 (2012).

[11] Z. Chen, S. Berciaud, C. Nuckolls, T. F. Heinz, and L. E. Brus, ACS Nano 4, 2964 (2010).

[12] H. Dong, W. Gao, F. Yan, H. Ji, and H. Ju, Anal. Chem. 82, 5511 (2010).

[13] P. Wang, T. Jiang, C. Zhu, Y. Zhai, D. Wang, and S. Dong, Nano Res. 3, 794 (2010).

[14] G. Konstantatos, M. Badioli, L. Gaudreau, J. Osmond, M. Bernechea, F. P. Garcia de Arquer, F. Gatti, and F. H. L. Koppens, Nat. Nanotech. 7, 363 (2012).
[15] Q. Bao and K. P. Loh, ACS Nano 3677 (2012).

[16] A. N. Grigorenko, M. Polini, and K. S. Novoselov, Nat. Photon. 6, 749 (2012).

[17] F. H. L. Koppens, D. E. Chang, and F. J. Garcia de Abajo, Nano Lett. 11, 3370-3377 (2011).

[18] A. Manjavacas, P. Norlander, and F. J. Garcia de Abajo, ACS Nano 6, 1724-1731 (2012).

[19] A. Manjavacas, S. Thongrattanasiri, D. E. Chang, and F. J. Garcia de Abajo, New. J. of Phys. 14, 123020 (2012).

[20] J. D. Cox, M. R. Singh, G. Gumbs, M. A. Anton, and F. Carreno, Phys. Rev. B 86, 125452 (2012).

[21] M. Feng, R. Sun, H. Zhan, and Y. Chen, Nanotechnology 21, 075601 (2010).

[22] Y. H. Lee, L. Polavarapu, N. Gao, P. Yuan, and Q.-H. $\mathrm{Xu}$, Langmuir 28, 321-326 (2012).

[23] D. Sarid and W. A. Challener, Modern introduction to surface plasmons: theory, Mathematica modeling, and applications (Cambridge; New York: Cambridge University Press, 2010).

[24] B. E. Kane, Phys. Rev. B 82, 115441 (2010).

[25] P. Hanarp, M. Käll, and D. S. Sutherland, J. Phys. Chem. B 107, 5768 (2003).

[26] F. Wang, Y. Zhang, C. Tian, C. Girit, A. Zettl, M. Crommie, and Y. R. Shen, Science 320, 206 (2008).

[27] Z. Q. Li, E. A. Henriksen, Z. Jiang, Z. Hao, M. C. Martin, P. Kim, H. L. Stormer, and D. N. Basov, Nat. Phys. 4, 532 (2008).

[28] P. Meystre and M. Sargent III, Elements of quantum optics, fourth edition (Springer-Verlag, Berlin Heidelberg, 
2007).

[29] D. A. Holm and M. Sargent III, Opt. Lett. 10, 405-407 (1985).

[30] M. O. Scully and M. S. Zubairy, Quantum optics (Cambridge: Cambridge University Press, 1997).
[31] R. W. Boyd, Nonlinear Optics, 3rd ed. (Academic, New York, 2008).

[32] T. Brunhes, P. Boucaud, S. Sauvage, F. Glotin, R. Prazeres, J.-M. Ortega, A. Lemaître, and J.-M. Gérard, Appl. Phys. Lett. 75, 835 (1999). 